$$
5 p_{1}+2 p_{3}+7 p_{4}=m+1, \quad 5 q_{1}+2 q_{3}+7 q_{4}=n .
$$

A solution of these equations is $p_{1}=4, p_{2}=3, p_{3}=11, p_{4}=7, p_{5}=1$, $q_{1}=4, q_{2}=3, q_{3}=11, q_{4}=7, q_{5}=3$. Hence a solution of (12) is $x=\alpha s^{4} t^{4}$, $y=\beta s^{3} t^{3}, \quad u=\lambda s^{11} t^{11}, \quad v=\mu s^{7} t^{7}, \quad w=\nu s t^{3} \quad$ where $s=a \alpha^{3} \lambda^{7} \nu+b \beta^{4} \mu^{11} \nu$, $t=c \alpha^{5} \lambda^{2} \mu^{7}$.

If $x=x^{\prime}, y=y^{\prime}, u=u^{\prime}, v=v^{\prime}, w=w^{\prime}$ is a given solution of (12) and the choice $\alpha=x^{\prime}, \beta=y^{\prime}, \lambda=u^{\prime}, \mu=v^{\prime}, \nu=w^{\prime}$ is made then $s=t$ and the solution becomes $x=x^{\prime} t^{8}, y=y^{\prime} t^{6}, u=u^{\prime} t^{22}, v=v^{\prime} t^{14}, w=w^{\prime} t^{4}$ which is equivalent to the given solution provided $t \neq 0$.

United States Naval Academy and

UNIVERSITY OF HOUSTON

\title{
VECTOR ANALOGUES OF MORERA'S THEOREM
}

E. F. BECKENBACH

Let the vector

$$
X \equiv X\left(x_{1}, x_{2}, x_{3}\right) \equiv X(x) \equiv X_{1} i+X_{2} j+X_{3} k
$$

be defined and continuous in the domain (non-null connected open set) $D$. Consider the mean-value vector

$$
\boldsymbol{X}^{(\rho)}(x) \equiv \frac{1}{\left|V_{\rho}\right|} \int_{V_{\rho}} \boldsymbol{X}(x+\xi) d V,
$$

where $V_{\rho}$ denotes the sphere

and $\left|V_{\rho}\right|$ its volume,

$$
\xi_{1}^{2}+\xi_{2}^{2}+\xi_{3}^{2}<\rho^{2},
$$

$$
\left|V_{\rho}\right| \equiv 4 \pi \rho^{3} / 3 \text {. }
$$

The vector (1) can be defined thus for only a part $D_{\rho}$ of $D$, but this is of no consequence since $\rho$ is arbitrarily small.

Since $\boldsymbol{X}(x)$ is continuous, it follows that $\boldsymbol{X}^{(\rho)}(x)$ has continuous partial derivatives of the first order; these are given by

$$
\frac{\partial}{\partial x_{p}} \boldsymbol{X}^{(\rho)}(x)=\frac{1}{\left|V_{\rho}\right|} \int_{S_{\rho}} \boldsymbol{X}(x+\rho \alpha) \alpha_{p} d \sigma,
$$

where $S_{\rho}$ denotes the surface of $V_{\rho}$ and $\alpha_{1}, \alpha_{2}, \alpha_{3}$ are the components of the unit vector along the outer normal to $S_{\rho}$.

Presented to the Society, September 5, 1941 under the title Vector formulations of Morera's theorem; received by the editors March 3, 1942. 
Mean-value functions are particularly useful in giving sufficiency proofs involving lessening of differentiability conditions. We shall use mean-value vectors to establish two generalizations of Morera's theorem to vector functions.

A vector $X(x)$ having continuous partial derivatives of the first order in the finite domain $D$ has been called $^{1}$ a Newtonian vector provided it is both irrotational and solenoidal; that is, provided its curl and its divergence both vanish identically in $D$ :

$$
\begin{aligned}
\operatorname{curl} X \equiv \nabla \times X \equiv & \left(\frac{\partial X_{3}}{\partial x_{2}}-\frac{\partial X_{2}}{\partial x_{3}}\right) i+\left(\frac{\partial X_{1}}{\partial x_{3}}-\frac{\partial X_{3}}{\partial x_{1}}\right) j \\
& +\left(\frac{\partial X_{2}}{\partial x_{1}}-\frac{\partial X_{1}}{\partial x_{2}}\right) k=0
\end{aligned}
$$

$$
\operatorname{div} X \equiv \nabla \cdot X \equiv \frac{\partial X_{1}}{\partial x_{1}}+\frac{\partial X_{2}}{\partial x_{2}}+\frac{\partial X_{3}}{\partial x_{3}}=0 .
$$

It follows readily ${ }^{2}$ that every Newtonian vector is a harmonic vector.

For a vector of components $X_{1}\left(x_{1}, x_{2}\right), X_{2}\left(x_{1}, x_{2}\right)$, equations analogous to (3) and (4) are the Cauchy-Riemann differential equations

$$
\frac{\partial X_{2}}{\partial x_{1}}=\frac{\partial X_{1}}{\partial x_{2}}, \quad \frac{\partial X_{2}}{\partial x_{2}}=-\frac{\partial X_{1}}{\partial x_{1}}
$$

for the function

$$
f(z) \equiv X_{2}\left(x_{1}, x_{2}\right)+i X_{1}\left(x_{1}, x_{2}\right), \quad z \equiv x_{1}+i x_{2} .
$$

This fact is taken as justification for considering Newtonian vectors as a generalization of analytic functions of a complex variable.

Let the vector $\boldsymbol{X}(x)$ have continuous partial derivatives of the first order in $D$. By Stokes' theorem, $\boldsymbol{X}(x)$ is irrotational if and only if for each reducible ${ }^{3}$ closed curve $C$ in $D$,

$$
\int_{C} X \cdot d R=0
$$

where $R$ is the vector from the origin to a moving point on $C$. By

${ }^{1}$ D. G. Fulton and G. Y. Rainich, Generalizations to higher dimensions of the Cauchy integral formula, American Journal of Mathematics, vol. 54 (1932), pp. 235241.

2 See, for instance, H. B. Phillips, Vector Analysis, New York, 1933, pp. 149, 153.

${ }^{3} \mathrm{~A}$ closed curve (or surface) in $D$ is reducible provided it is rectifiable (or of finite area) and can be shrunk continuously in $D$ to a point of $D$. The forms of Stokes' theorem and Green's theorem referred to in this paragraph may be found in H. B. Phillips, loc. cit., pp. 62, 68, 72. 
Green's theorem, $\boldsymbol{X}(x)$ is irrotational if and only if for each reducible closed surface $S$ in $D$,

$$
\int_{S} n \times X d \sigma=0
$$

where $n$ is the unit vector along the outer normal to $S$; and again by Green's theorem, $\boldsymbol{X}(x)$ is solenoidal if and only if for each reducible closed surface $S$ in $D$,

$$
\int_{S} n \cdot X d \sigma=0
$$

It follows that Newtonian vectors are characterized by (5) and (7), and also by (6) and (7); but (cf. Morera's theorem) the assumption that $\boldsymbol{X}(x)$ has continuous partial derivatives of the first order now is redundant, ${ }^{4}$ as we shall show. We shall further lessen the sufficiency conditions by replacing the integral conditions (5), (6) and (7) by weaker local conditions which are implied by (5), (6) and (7), respectively.

We shall denote by $S(x, r)$ the sphere with center $(x)$ and radius $r$, and by $C_{1}(x, r), C_{2}(x, r), C_{3}(x, r)$ the circles with center $(x)$ and radius $r$ in planes perpendicular to the $x_{1}, x_{2}, x_{3}$ axes, respectively. Let

$$
C(x, r) \equiv C_{1}(x, r) i+C_{2}(x, r) j+C_{3}(x, r) k,
$$

and let $o\left(r^{t}\right)$ denote a function (not always the same function) of $r$ such that

$$
\lim _{r \rightarrow 0} \frac{o\left(r^{t}\right)}{r^{t}}=0 .
$$

THEOREM 1. Let the vector $X(x)$ be continuous in the finite domain $D$. If

$$
\int_{\mathrm{C}_{(x, r)}} \boldsymbol{X} \cdot d \boldsymbol{R}=o\left(\boldsymbol{r}^{2}\right)
$$

and

$$
\int_{S(x, r)} n \cdot X d \sigma=o\left(r^{3}\right)
$$

uniformly in each sphere in $D$, then $X(x)$ is a Newtonian vector.

${ }^{4}$ See H. B. Phillips, loc. cit., pp. 177, 178, for a proof of the result that if the continuous vector function $\boldsymbol{X}(x)$ satisfies (5) and (7), then $\boldsymbol{X}(x)$ is a Newtonian vector. 
Proof. Since (8) and (9) hold uniformly in each sphere in $D$, we have

and

$$
\frac{1}{\left|V_{\rho}\right|} \int_{V_{\rho}}\left(\int_{C_{(x, r)}} \boldsymbol{X} \cdot d \boldsymbol{R}\right) d V=o\left(r^{2}\right)
$$

$$
\frac{1}{\left|V_{\rho}\right|} \int_{V_{\rho}}\left(\int_{S(x, r)} n \cdot X d \sigma\right) d V=o\left(r^{3}\right)
$$

in $D_{\rho}$. Interchanging the order of integration, we obtain

$$
\int_{C_{(x, r)}} \boldsymbol{X}^{(\rho)} \cdot d \boldsymbol{R}=o\left(r^{2}\right)
$$

and

$$
\int_{S(x, r)} n \cdot X^{(\rho)} d \sigma=o\left(r^{3}\right)
$$

Since $\boldsymbol{X}^{(\rho)}(x)$ has continuous partial derivatives of the first order in $D_{\rho}$, about any point $\left(x^{(0)}\right)$ in $D_{\rho}$ we have the finite Taylor development

$$
\begin{aligned}
\boldsymbol{X}^{(\rho)}(x)=\boldsymbol{X}^{(\rho)}\left(x^{(0)}\right) & +\frac{\partial \boldsymbol{X}^{(\rho)}}{\partial x_{1}}\left(x_{1}-x_{1}^{(0)}\right)+\frac{\partial \boldsymbol{X}^{(\rho)}}{\partial x_{2}}\left(x_{2}-x_{2}^{(0)}\right) \\
& +\frac{\partial \boldsymbol{X}^{(\rho)}}{\partial x_{3}}\left(x_{3}-x_{3}^{(0)}\right)+o(r)
\end{aligned}
$$

where the partial derivatives are evaluated at $\left(x^{(0)}\right)$. Computations yield

$$
\int_{C_{\left(x^{(0)}, r\right)}} \boldsymbol{X}^{(\rho)} \cdot d R=\pi r^{2} \operatorname{curl} \boldsymbol{X}^{(\rho)}\left(x^{(0)}\right)+o\left(r^{2}\right)
$$

and

$$
\int_{S\left(x^{(0)}, r\right)} n \cdot X^{(\rho)} d \sigma=(4 / 3) \pi r^{3} \operatorname{div} X^{(\rho)}\left(x^{(0)}\right)+o\left(r^{3}\right) .
$$

Now (10)-(13) give

$$
\operatorname{curl} \boldsymbol{X}^{(\rho)}(x)=0
$$

and

$$
\operatorname{div} \boldsymbol{X}^{(\rho)}(x)=0
$$


throughout $D_{\rho}$; that is, $\boldsymbol{X}^{(\rho)}(x)$ is a Newtonian vector. Hence $\boldsymbol{X}(x)$, the uniform limit of $\boldsymbol{X}^{(\rho)}(x)$ as $\rho \rightarrow 0$, is a Newtonian vector.

THEOREM 2. Let the vector $X(x)$ be continuous in the finite domain $D$. If

and

$$
\int_{S(x, r)} n \times X d \sigma=o\left(r^{3}\right)
$$

$$
\int_{S(x, r)} n \cdot X d \sigma=o\left(r^{3}\right)
$$

uniformly in each sphere in $D$, then $X(x)$ is a Newtonian vector.

Proof. The proof is analogous to that of Theorem 1, with

$$
\int_{S\left(x^{(0)}, r\right)} n \times X^{(\rho)} d \sigma=(4 / 3) \pi r^{3} \operatorname{curl} X^{(\rho)}\left(x^{(0)}\right)+o\left(r^{3}\right)
$$

in place of (12).

The University of Michigan 